\title{
Rupture et continuité : le futur de la tradition en muséologie
}

\section{Bruno Brulon Soares}

Traducteur : Marion Bertin

\section{OpenEdition \\ Journals}

\section{Édition électronique}

URL : http://journals.openedition.org/iss/1963

DOI : $10.4000 /$ iss. 1963

ISSN : 2306-4161

\section{Éditeur}

ICOM - International Council of Museums

\section{Édition imprimée}

Date de publication : 1 août 2020

Pagination : 29-42

ISBN : 978-2-491997-11-3

ISSN : 2309-1290

\section{Référence électronique}

Bruno Brulon Soares, «Rupture et continuité : le futur de la tradition en muséologie », ICOFOM Study Series [En ligne], 48-1 | 2020, mis en ligne le 01 août 2020, consulté le 08 août 2020. URL : http:// journals.openedition.org/iss/1963; DOI : https://doi.org/10.4000/iss.1963 


\section{Introduction}

\section{Rupture et continuité : \\ le futur de la tradition en muséologie}

\section{Bruno Brulon Soares \\ Universidade Federal do Estado do Rio de Janeiro, UNIRIO}

\section{Traduction de Marion Bertin}

Récemment au Museu do Índio, une institution nationale à Rio de Janeiro sauvegardant une importante part du patrimoine brésilien autochtone, les conservateurs ont discuté une nouvelle méthode pour la conservation des collections ethnographiques. D'après un savoir local, les professionnels de musée ont considéré d'utiliser un pédoncule de banane émincé comme nouvelle technique pour le contrôle des nuisibles dans une des réserves les plus sensible du musée. Selon l'un des conservateurs, cette méthode aide à empêcher l'infestations des matériaux organiques en alertant le personnel de la présence d'insectes. Le pédoncule a pour but d'attirer les insectes et les autres nuisibles avant qu'ils n'atteignent les colletions, une méthode adoptée par quelques musées en Amérique latine comme solution précaire aux problèmes d'infestation qui sont particulièrement dangereux pour les objets constitués de matériaux organiques des collections ethnographiques.

Alors que les manuels de conservation auraient recommandé une approche plus «traditionnelle » face au problème d'infestation, la tradition elle-même nécessitait d'être réinventée dans ce cas. Le musée se tourne vers des savoirs alternatifs, change ses méthodes en l'absence d'autres ressources disponibles. Tandis que certaines grandes institutions peuvent s'appuyer sur des technologies de pointe pour la conservation de leurs collections, de nombreux musées, en particulier dans les zones tropicales, doivent adapter leur rôle traditionnel de simple représentation d'une mémoire nationale collective (Smeds, 20I9) afin de trouver des manières alternatives de résoudre les enjeux contemporains. Dans l'exemple du Museu do Índio, une institution avec une longue participation historique des communautés autochtones - pour l'exposition, la documentation et la conservation -, ce qui est nouveau ou « moderne » est l'introduction d'un savoir local pour le meilleur fonctionnement de ce musée, enraciné dans la tradition européenne dès sa création. 
Mais que sont « la tradition » et « la modernité » dans la muséologie ? Pour répondre à cette question quelque peu rhétorique, nous avons besoin de comprendre comment le musée et la muséologie traitent du temps. En réalité, l'opposition dépassée entre «moderne » et «traditionnel », définie de manière si rigide en Occident, a montré ses limites pour expliquer le rôle et les fonctions des musées dans le temps présent. Cependant, personne ne peut ignorer qu'une perception linéaire du temps en tant que marche vers le progrès a marqué l'histoire des musées depuis le Siècle des Lumières et la colonisation.

Comme l'a noté l'anthropologue Johannes Fabian, « le temps peut donner forme à des relations de pouvoirs et d'inégalité », ce qui est le cas de l'évolutionnisme social et de ses expressions matérielles dans les musées. Le temps linéaire, en tant que construction philosophique adoptée par les sciences sociales depuis le XIX ${ }^{e}$ siècle, exerce toujours une influence sur les sociétés par le fait des conditions de la production industrielle capitaliste (Fabian, 2002 [I983], p. IX). Selon la "politique du temps », les relations de pouvoir sont construites au sein d'une définition de ce qui est traditionnel, primitif et sous-développé en comparaison au monde moderne, civilisé et développé. Ainsi, une définition du temps et la catégorisation hiérarchique de l'humanité qui s'en suit sont fondamentales pour l'anthropologie ainsi que pour les musées «modernes ».

La «tradition », en tant que notion fondée sur un temps linéaire, a été utilisée pour affirmer les différences en tant que distances temporelles et spatiales. Cela implique une séparation symbolique entre le passé et le présent ou entre les différentes sociétés et populations assignées à des places différentes sur l'échelle du temps du progrès et de la civilisation. En quelque sorte, la tradition est reliée à l'« authenticité », un concept également lié à l'origine des musées. L'idée d'un passé authentique (sauvage, tribal ou paysan), selon Fabian, sert à dénoncer le «présent inauthentique (sans racine, évolué, acculturé) 》(2002 [I983], p.II). Une telle conception aide à définir une rupture dans le temps qui situe dans différents espaces le sujet de science et ses objets d'études.

La muséologie, d'une certaine manière, a hérité de cette perception préconçue de la différence culturelle et des diverses formes de savoir selon un temps linéaire, inventant ainsi ses propres traditions d'après la conception occidentale du temps philosophique, matérialisée dans les représentations muséales de l'Autre au sein de dioramas voulus «traditionnels».

La muséologie théorique n'est pas exempte d’un tel héritage, revu dans des essais critiques contemporains. Lidéologie du développement est largement exprimée dans la théorie muséologique produite dans les années i980. Par exemple, une forme de progrès, d'amélioration et de développement est délibérément appliquée au discours muséologique lors du symposium annuel d'ICOFOM en I988, tenu en Inde, lors duquel le thème débattu est « Muséologie et pays en voie de développement - aide ou manipulation?». Comme le souligne Vinoš Sofka dans son étude parue l'année suivante, les débats sur le « développement des sociétés » portent non seulement sur « un point de vue historique dans le 
respect des expériences passées dans les différents pays, mais également sur les changements actuels, accélérés et plus complexes à travers le monde » (Sofka, I989, p. I3). Au fil des années, les auteurs d'ICOFOM défient le point de vue prévalant considérant qu'une personne venant d'un pays « sous-développé » ne peut produire aucune forme significative de pensée théorique car la théorie et la science sont principalement produites dans le Nord mondialisé.

En insistant sur l'importance du temps linéaire dans la prédiction d'une muséologie future, le thème proposé pour le symposium d'ICOFOM en I989 est «La prospective - un outil muséologique? Muséologie et futurologie». Ici, la futurologie, la science ayant pour but de prédire l'avenir, ne peut ignorer son caractère autoritaire et le principe ethnocentrique qui la sous-tend, défend Waldisa Rússio dans son approche critique du thème. L'auteure brésilienne condamne la futurologie comme outil de manipulation orienté en faveur des intérêts de groupes et États-nations spécifiques, «surtout les plus riches » (1989, pp. 219-20).

Aujourd'hui, ICOFOM n'a plus les mêmes visions des sociétés et des musées - et du temps lui-même - par rapport à celles exprimées dans les années i980. Nous sommes passés dans un autre temps de production théorique, qui reconnait que l'innovation peut s'exprimer dans la réinvention de pratiques traditionnelles dans la vie quotidienne d'un musée, tel que l'usage du pédoncule de banane comme méthode de conservation. Les savoirs autochtones et la participation de communautés sont aujourd'hui d'importantes dimensions de la tradition muséale, mais tous impliquent la possibilité constante de changement et d'adaptation dans le but de répondre à des demandes contemporaines et de faire de la décolonisation une pratique en cours.

Le thème central de la Conférence Générale de Kyoto (Japon) en 2org, « Les musées comme hubs culturels : le futur de la tradition », est interprété comme une incitation contemporaine à reconsidérer le passé en vue du futur. Les débats d'ICOFOM à Kyoto montrent de manière évidente les implications du temps dans les études des traditions muséologiques interrogeant ces traditions ellesmêmes et leurs possibles futurs. Comme le montrent de nombreux articles de ce numéro, même quand une rupture avec le temps linéaire est décidée, dévoilant la tradition occidentale comme fondation des musées, il n'est jamais facile de laisser de côté des éléments du passé qui sont primordiaux dans notre idée de la continuité. La Conférence de Kyoto est un bon exemple qui montre que changer le futur nécessite d'abord et avant tout de reconnaître les traditions qui nous ont menés au présent, avant de trouver un moyen de poursuivre.

Dernièrement, le musée est entré dans une époque où nous réalisons qu’il est nécessaire de subvertir la tradition, de passer à des anti-manuels de muséologie (Zavala, 20I2) et d'assouplir les définition et règles strictes qui ne s'appliquent pas à l'immédiate réalité. Comme le note Homi Bhabha, la nouveauté des pratiques culturelles et des récits historiques pénètre le monde par la fragmentation et l'hybridité. Ce processus demande la reconnaissance d'un « espace 
entre-deux », dont le sujet décentré est signifié «dans la temporalité agitée du transitionnel ou du provisoire dans le présent » (I994, p. 216). Le présent est ainsi réinventé entre tradition et innovation et c'est à travers cette contradiction de la tradition que les musées, en quelque sorte, trouvent un moyen de se transformer pour le futur.

Ce numéro des ICOFOM Study Series ne traite donc pas d'une vision prospective du futur, ni n'aborde notre démarcation par rapport au passé. En s'intéressant au «futur de la muséologie », il traite de ce qui peut être créé dans l'espace entre tradition et nouveauté, auquel est lié le potentiel d'innovation et d'improvisation pour le travail quotidien du musée et constitue une tendance de la muséologie dans le présent.

\section{Tradition et l’idée de continuité}

Dans une approche rhétorique, la «tradition » peut être entendue comme un ensemble de techniques développées pour permettre aux anciens orateurs, récitant sans recours à un manuscrit, d'inventer, d'organiser et de se remémorer les points principaux et les arguments d'un discours et d'engager des discussions collectives (Yates, 1966; Fabian, 2002 [1983]). Dans cette perspective, la tradition est un discours à propos du passé qui sert à donner du sens aux expériences présentes en créant un effet de continuité. Dans le passé, les «traditions » dans les musées se réfèrent aux cultures populaires (Rivière \& Cuisenier, 1972) ; la notion de «populations traditionnelles » est toujours communément employée pour désigner les populations autochtones ou les Afro-descendants vivant dans des parties du monde prétendu contemporain (Cunha \& Almeida, 2009).

Cependant, dans le domaine de la muséologie, le «musée traditionnel » est une expression souvent employée pour désigner un modèle obsolète qui ne répond plus aux besoins des sociétés actuelles. Ainsi, «tradition » en muséologie se réfère à la Modernitér. La forme traditionnelle du musée, en tant qu’institution reposant sur des artefacts matériels servant les intérêts de l'État moderne, est une importante part des imaginaires dont nous avons hérités et une influence des études muséologiques aujourd'hui. La muséologie est traditionnelle dans son essence, dans sa définition superficielle donnée au début du XXe siècle comme «science de l'organisation des musées » (Augé, I93I, p.I048), jusqu'au développement d'expressions plus réflexives dans les décennies suivantes.

En tant qu'institution "moderne », le musée est plus récemment perçu par la muséologie comme « un acte de violence, une rupture avec les traditions » dans certaines sociétés où le temps n'était pas défini selon la logique européo-centrique matérialisée par les collections de musées (Konaré, I987, p.I5I).

\footnotetext{
I. La Modernité entendue ici comme la consolidation des empires coloniaux en Europe et ses conséquences, mais aussi l'assujettissement de populations et de cultures dans leurs combats pour la libération, en tant que leur histoire est écrite et narrée principalement par le biais du discours colonial produit dans le cadre de ce pouvoir (Mignolo, 1995).
} 
Les études historiques mettent en valeur la place de la Modernité au sein des musées et du patrimoine culturel, en parallèle de leurs conséquences dans les différentes parties du monde (Poulot, I998; Pearce, 20I0; Mairesse, 20II), ce qui résulte du développement d'une théorie plus critique. Les études récentes critiquent la colonialité de la tradition muséologique (Brulon Soares \& Leshchenko, 20I8) et traitent des différentes formes de subversion et de dépassement du passé colonial, comme dans les études présentées dans cette édition par Bertin et Graff ainsi que dans l'analyse de Mellado \& Andrade à propos d'une «muséologie métisse ».

Comme le montrent des contributeurs de ce numéro, la muséologie réflexive et la théorie critique sont fondamentales pour la décolonisation de cette discipline académique. Par l'analyse des musées nationaux dans les jeunes États du Pacifique, Marion Bertin démontre de quelle manière le musée, en tant qu'héritage occidental, est adapté dans ces sociétés, en impliquant des communautés et des groupes autochtones pour la représentation de la nation. Bertin défend que les musées dans les îles du Pacifique, un héritage douloureux de l'époque coloniale, peuvent être réinterprétés comme une forme de tradition utile fonctionnant en tant qu'instrument politique pour reconstruire le futur. En tant qu'outil d'affirmation politique, les musées dans les îles du Pacifique sont maintenant transformés grâce à une interprétation post-coloniale de leurs pratiques traditionnelles, en impliquant des membres de communautés dans la conservation des collections et dans la transmission du patrimoine culturel. En se connectant à un héritage ancien matérialisé dans les collections pré-coloniales acquises par les Occidentaux, ces musées sont redéfinis dans le présent en tant qu'espaces de négociation des références du passé.

Une appropriation similaire du dispositif muséal est décrite par Julie Graff dans son analyse de l'exposition Öndia'tahterendih, oubliées ou disparues: Akonessen, Zitya, Marie et les autres (Forgotten or Missing), présentée au Musée de la Civilisation du Québec en 2018 sous le commissariat de Sylvie Paré. L'exposition traite des féminicides de femmes autochtones au Canada en présentant le travail de dix artistes honorant la mémoire de femmes autochtones tuées, en cherchant à engager émotionnellement le public et, dans le même temps, à développer une conscience politique à propos d'un sujet sensible et souvent oublié dans les musées. Cet exemple d'exposition qui dénonce les violences contre les femmes autochtones en exposant les racines du colonialisme par le biais de l'art montre franchement comment les musées peuvent être utilisés pour questionner la tradition et exposer le passé en vue de transformer le futur.

En prenant une distance avec les interprétations traditionnelles du passé colonial par une approche anti-coloniale, ces études tentent de prouver que la tradition est une part logique du présent et peut être remaniée par les musées pour le bénéfice des sociétés et des groupes persécutés. En effet, cela peut indiquer aux lecteur.rice.s à quel point la pensée muséologique a été transformée depuis les premières générations de penseurs d'ICOFOM et les critiques du colonialisme, qui ont perçu le musée comme « la sanction d'une 
blessure ouverte, la conséquence d'une désagrégation des structures sociales traditionnelles » (Konaré, 1987, p. 151), d'après les mots du politicien malien Alpha Omar Konaré, un nom important pour notre tradition.

Tandis que certaines approches face au colonialisme peuvent proposer une rupture frontale, d'autres envisagent la possibilité d'une hybridité entre les structures du passé et les changements du présent. Leonardo Mellado et Pablo Andrade envisagent le cadrage théorique et la définition de la muséologie eux-mêmes comme partie de l'héritage, qui s'est révélé être varié, hybride ou métisse dans une perspective post-coloniale. Les auteurs suggèrent le concept de «muséologie métisse », d'après une réflexion critique du Museo Histórico Nacional of Chile et son exposition Museo Mestizo: Fundamentos para el cambio de guión organisée en 20I8. Le caractère métissé de la muséologie post-coloniale se réfère à un patchwork conceptuel et dynamique qui permet la reconnaissance et la validation de diverses sources théoriques et cadres méthodologiques.

Dans la présente muséologie métisse, le passé et le futur sont mélangés et imbriqués pour réinterpréter les pratiques et procédures muséales fondamentales. Les fonctions traditionnelles du musée, telles que collecter, conserver et exposer, sont exercées avec un éclairage nouveau pour de nouveaux buts. D'après cet exemple, nous devons poursuivre notre réflexion sur les acceptions différentes de la tradition et du «traditionalisme ». En prenant lui-même de la distance avec le traditionalisme, le musée peut devenir métisse en incluant de nouveaux acteurs pour légitimer leur patrimoine culturel - tel que le patrimoine autochtone, le patrimoine des diasporas africaines ou le patrimoine paysan. Mellado \& Andrade attirent l'attention sur le fait que le métissage est peu représenté (ou célébré) dans la mémoire officielle. Ainsi, surgit le besoin d'une nouvelle configuration du musée en tant que champ de bataille pour de nouvelles identités et pour des identités indéfinies et diverses non matérialisées dans des collections d'objets.

Dans sa propre interprétation d'une muséologie variée, Graff défend que le processus de décolonisation du musée ne peut être accompli sans le travail d'intellectuels et praticiens autochtones - une idée qui n'est toujours pas une tradition pour des institutions qui reproduisent les exclusions ainsi que la colonialité du pouvoir (Quijano, 200o), alors même quelles parlent de « décolonisation » d'après un point de vue européo-centrique. Comme ces auteurs le montrent, il n'y a pas de transition vers le futur sans friction entre le passé et le présent, ce qui implique la reconnaissance de la tradition comme partie importante de l'héritage muséologique.

Au cours des dernières décennies, depuis les interprétations les plus critiques du musée, la muséologie a développé une nouvelle tradition réflexive exprimée par les publications d'ICOFOM et par la mise en place d'une « théorie de la muséologie ». François Mairesse, dans son introduction à ce numéro, interprète la collecte de pratiques, représentations, expressions et savoirs qui constituent la muséologie en tant que patrimoine immatériel transmis par le biais de géné- 
rations de penseurs, au-delà de ce qui est organisé dans des textes et manuels publiés. Il défend que la muséologie transmet un corpus méthodologique qui, à travers le temps, a produit une forme de discipline académique cohérente avec ses valeurs partagées et ses défis particuliers pour le futur.

Les auteurs de ce numéro - ainsi que son éditeur - ont appris à articuler leur pensée muséologique en prenant en compte ce patrimoine immatériel qui leur a été confié par les générations passées. ICOFOM est né dans les années I970 comme un comité réflexif questionnant les principes du savoir muséal produit au sein de la communauté de l'ICOM. Aujourd'hui, après quelques générations de théoriciens et de chercheurs ayant laissé leur héritage, nous pouvons nous retourner vers leurs travaux pour y trouver un témoignage. En scrutant leur textes et réflexions passées, la principale leçon à tirer peut être de réaliser qu'une pensée critique implique de questionner les fondements de n'importe quel savoir acquis. Mais une telle leçon réflexive demande du temps pour être acquise et générer une tradition par elle-même. Ce numéro des ICOFOM Study Series est en cela un témoignage pour une nouvelle génération de penseurs qui ont appris la leçon de la méta-muséologie en questionnant leur propre héritage et en subvertissant la tradition pour créer leur propre muséologie.

\section{Ruptures et changements : une idée de nouveauté}

Depuis la première vague de nouveauté dans la tradition muséologique, le mouvement de Nouvelle Muséologie dans les années i970 et 1980, la muséologie a embrassé une approche critique du musée dans sa forme européo-centrée héritée du XIX siècle (Varine, 20I7). Ceci inaugure une rupture avec le «musée traditionnel » permettant la reconnaissance de nouvelles expériences ayant en commun une ouverture vers la différence culturelle et la participation sociale sans précédent dans l'histoire de la muséologie.

La nouvelle muséologie en tant que mouvement international, absorbée comme une "nouvelle tradition de la muséologie » ou un « retour aux sources de la muséologie » (Devallées, I992, p. 22-23) établit une interprétation du temps en divisant l'histoire de cette jeune discipline entre l'ancien et le nouveau, sur la base d'une transformation radicale des pratiques et idéologies muséales. La «muséologie (nouvelle)», comme la conçoit André Desvallées et se l'approprient d'autres penseurs réflexifs, propose une rupture avec la tradition dans un monde qui est alors désigné comme « post-colonial » ou «post-moderne » - les nouveaux termes devenus depuis hégémoniques dans les sciences sociales.

En abordant la multiplication des différences culturelles liée à la crise globale des valeurs qui suit le déclin des utopies modernes et les processus de décolonisation, la nouvelle muséologie est née comme une promesse de rupture avec le récit européen universel du progrès et de la civilisation. Cependant, il demeure la forme de narration du monde des musées en termes de pratiques «nouvelles » et « anciennes » et applique à la muséologie la division géopolitique entre pays développés et pays en voie de développement. Comme la 
Déclaration de Québec de 1984 le statue : « la nouvelle muséologie [...] est premièrement et principalement concernée par le développement des populations, reflétant les principes modernes qui ont conduit leur évolution, tout en les associant simultanément aux projets pour le futur...» (je souligne). Comme nous le voyons, l'attitude paternaliste vis-à-vis des musées fondés par des communautés est explicitement inspirée par le principe évolutionniste, qui définit le développement comme un objectif à atteindre pour les populations en voie de développement. En certains contextes, comme en Amérique latine, la nouvelle muséologie et l'écomuséologie transposent une hiérarchie de pouvoir fondée sur la centralisation de l'État à des pratiques expérimentales hors du champ de la muséologie traditionnelle - en périphérie du champ muséal établi.

En appliquant une logique développementaliste des États-nations au contexte des musées dans le Sud mondialisé, la nouvelle muséologie souligne la division entre les pays industrialisés, les États communistes et le Tiers Mondes établie entre les années 1950 et 1970 quand émerge un nouvel ordre (hiérarchique) mondial (Pletsch, I98I; Mignolo, I995). Ainsi, le discours sur la décolonisation de la muséologie ne rompt pas avec la reproduction de la structure capitaliste du pouvoir fondée sur une distribution inégale des ressources en conservant la désignation ancienne du Vieux Monde comme étant «purement moderne, un havre de science et de décision utilitaire, technologique, efficient, démocratique, libre » (Pletsch, I98I, p. 574).

Quelle nouvelle méthodologie utiliser pour mieux refléter le musée et ses expressions plurielles au XXI siècle? Pour Olivia Guiragossian, dans ce numéro, le futur de la muséologie est lié au futur des recherches sur les musées et le développement de nouveaux instruments pour les observer dans des sociétés en plein changements. Le focus de l'auteure est l'analyse des observations du musée elles-mêmes et les méthodes utilisées pour observer les différentes formes et expressions placées sous l'étiquette de «traditionnel». Un des premiers défis pour l'observation des musées et la production massive de données est la définition exacte et opérationnelle d'un musée, comme l'indique l'auteure.

Bien que, par ses racines, la nouvelle muséologie ne soit pas une déclaration délibérée contre les pratiques et théories « anciennes », dans certains cercles elle est interprétée comme une rupture avec la structure moderne du musée - notamment avec sa forme la plus traditionnelle, en tant que bâtiment avec des collections matérielles. Le résultat est de mettre en valeur l'opposition binaire entre musée orienté vers le public et musée attaché à la préservation des collections. La rupture artificielle entre ancien et nouveau produit une idée de nouveauté dans les pratiques qui considèrent le public comme une force déterminante pour les musées alors qu'il réitère l'imaginaire d'une muséologie datée. Plusieurs articles de ce numéro remettent en question le discours binaire produisant des oppositions artificielles dans le savoir muséologique. Dans son analyse rhétorique, Elizabeth Weiser défend que la dichotomie traditionnelle entre visiteur et objet, inscrite dans l'actuelle définition de l'ICOM, est devenue obsolète pour les approches les plus récentes, ce qui est rendu évident par 
l'analyse textuelle de la nouvelle définition du musée proposé par l'ICOM lors de son Assemblée Générale en 2org. En prenant elle-même distance avec une dichotomie entre "tradition » et "modernité », «ancien » et "nouveau », Weiser préfère considérer de manière critique la place du musée dans l'actuelle muséologie dans un sens plus nuancé et élargi.

D'autres recherches considèrent l'importance des études portant sur la culture matérielle et l'objet de musée comme une part de l'héritage de la muséologie. En réalité, les études sur l'objet de musée sont jusqu’à récemment centrales pour le patrimoine muséologique. Les analyses, telle que celle présentée par Nina Robbins dans ce numéro, mettent en valeur le besoin de poursuivre des recherches dans cette branche des études muséologiques. Robbins propose le concept d'« object energy » pour décrire l'information écrite entourant un objet spécifique dans un musée.

Dans une approche parallèle, Fabien Van Geert propose le renouveau de la recherche muséologique considérant le "géo-patrimoine »- le patrimoine géologique dans sa diversité. L'auteur note les défis théoriques pour la muséologie à propos de la conservation in situ et ex situ du patrimoine géologique en France. La valeur de l'article de Van Geert est d'attirer l'attention sur le manque d'études et de publications au sujet du géo-patrimoine dans les principales plateformes et revues muséologiques, un fait contrastant avec l'importante quantité de ce type de patrimoine dans les réserves de musées. Un des aspects mentionnés dans son analyse fait référence à la relation entre la théorie et la diffusion de nouvelles pratiques au sens muséologique (impliquant à la fois des musées avec des collections traditionnelles ex situ comme du patrimoine géologique conservé in situ).

Quand nous nous retournons vers les «nouvelles » interprétations de la muséologie maintenant devenues une part de notre tradition, nous ne pouvons ignorer les différentes forces en conflit pour définir le principal sujet des études muséologiques et des discours sur le musée. Les articles présentés ici aide à ouvrir quelques questions pour le futur, en considérant le temps comme une réinvention constante. Quelles sont les traditions que nous voulons conserver et transmettre pour les pratiques et réflexions futures? Quelles parties du passé devons-nous garder et quelles parties est-on prêts à laisser de côté ? Ces questions dépendent de la « décomposition du temps »- telle que Bhabha la décrit - pour trouver une réponse et demandent la reconnaissance de la muséologie en tant que discipline agissant dans les espaces d'entre-deux, connectant l'« ancien » et le «nouveau», où les traditions peuvent être renégociées avant d'être transmises aux futures générations.

\section{Transition vers le futur - une fois de plus}

Le savoir est toujours fait de discours variés, hétérogènes et souvent contradictoires. De plus, dans n'importe quel groupe ou communauté, l'affirmation de l'autorité sur le savoir impose une séparation entre la tradition et l'innovation, 
la légitimation de quelques références établies stables dans une nouvelle configuration altérée. À la suite des brûlants débats lors de la Conférence Générale de Kyoto en 2019, il n'est pas risqué de prédire que la muséologie est sur le point de réinventer ses traditions une fois de plus au XXI siècle.

Les musées d'aujourd'hui sont face à de nouveaux et imprévisibles défis qui donnent le ton pour une approche plus critique et plus expérimentale de la muséologie, dont la tradition est en cours de renégociation, tandis que nous reconsidérons nos affiliations au passé. Dépassant le souhait de prévoir le futur de la muséologie, ICOFOM tente, par ses symposiums et ses publications, de cartographier différentes approches de la pensée muséologique qui constituent notre présent. Anticiper le changement dans le monde des musées marqué par l'expansion des valeurs néo-libérales et par des crises économiques est un souci constant, tel qu'exprimé dans les débats de notre comité quelques années auparavant à propos des nouvelles tendances de muséologie (Mairesse, 2015) - dont quelques-unes sont aujourd'hui datées, comme je l'ai indiqué dans cette introduction.

Plus encore aujourd'hui qu'au cours des dernières années, nous vivons une période de grande incertitude pour les musées et pour les réflexions muséologiques. Ce numéro s'inscrit parmi l'histoire des publications lancées en pleine pandémie de COVID-rg, alors que la majorité des institutions culturelles à travers le monde sont fermées au public et que les effets d'une longue crise économique submergent déjà le champ muséal, avec des licenciements de masse dans quelques institutions centrales et des coupures de salaire pour les professionnels de musées les plus vulnérables. Alors que le futur des musées est relégué au cyberespace - une tendance du discours actuel pendant la pandémie - ce numéro des ISS ne traite pas de ce futur virtuel prédit. La plupart des contributeurs de cette publication choisissent de discuter des implications politiques du changement en lien avec les récents appels pour des représentations sociales, qui amènent de nouveaux enjeux pour les musées et pour l'interprétation du patrimoine culturel.

Mais la pandémie actuelle évince en réalité quelques symptômes des transformations mondiales des sphères culturelles, politiques et économiques, préparées bien avant le développement du coronavirus. Alors que les centres urbains mondiaux, de Paris à Rio de Janeiro, font face à l'apparition de "minorités visibles » et à une pluralité de manière de vivre en société (Bancel et al, zoro, p. Io), la démocratie culturelle en tant qu'idéal est confrontée à une fragmentation de l'espace public et au développement de différences et d'inégalités. Les musées doivent faire face à une urgente crise de la représentation due à des patrimoines non-linéaires et polysémiques et au discours sur la décolonisation, qui incluent des demandes en faveur d'une «queerisation » des collections et des pratiques, tandis qu'ils doivent s'adapter aux changements économiques accélérés, entraînant une précarité du travail dans l'intégralité du secteur culturel. 
Avant l'imminence d'une nouvelle crise du musée, quelques questions peuvent être posées quant au futur de la muséologie : comment nos institutions culturelles peuvent-elles lutter contre la nouvelle réalité économique et rester pertinentes pour les sociétés dans le futur? Comment les musées peuvent-ils opérer une transition vers le numérique tout en trouvant des ressources en étant privés de publics physiques? Les musées auront-ils toujours les moyens et le personnel pour conserver leurs collections matérielles dans d'immenses réserves tout en communiquant et rassemblant de nouveaux publics en ligne? Pour les musées des quatre coins du monde, les problèmes actuels liés à l'économie culturelle provoquent un débat urgent en vue de redéfinir des priorités. Il est possible que la muséologie, en tant que discipline traitant des valeurs sous-jacentes de l'institution muséale, comme le suggère Robbins, n'ait jamais été aussi précieuse pour le futur des musées - un futur qui ne pourra dépendre que des traditions que nous décidons de transmettre maintenant.

Alors que j'écris cette introduction, les notions même de «modernité » et de «tradition » sont en plein changement. Avançant dans le futur, les musées peuvent nécessiter de se réinventer, dans des sociétés où le temps est en train d'être réécrit et re-présenté sous de nouvelles formes, par la manipulation des faits sociaux, dans le cadre d'un nouvel agenda politique pour le XXI eiècle. Dans son article de cette édition, Luciana Menezes de Carvalho s'intéresse au rôle de la tradition dans l'ère de «post-vérité » dans laquelle le temps est renégocié pour des raisons politiques actuelles. En tant que part importante de la modernité, les musées sont mis au défi face à des discours négationnistes qui mettent en doute la science et affaiblissent le rôle d'institutions centrales dans le fonctionnement de sociétés démocratiques. La nouvelle configuration des États-nations néo-libéraux et leur pouvoir politique inventent de nouvelles traditions, diffusées par les réseaux sociaux comme une promesse de futur qui ne nécessite ni la science ni les scientifiques pour exister - un fait que la pandémie de COVID-rg amène à sérieusement questionner.

La pensée critique ne doit pas impliquer la négation du passé. Au contraire, elle dépend d'un regard vers nos traditions et d'une reconnaissance de leur existence, avec un point de vue critique. Comme Carvalho le présente dans son travail, la science est définie de manière politique et la muséologie a été ellemême définie comme une discipline scientifique fondée sur une tradition de recherches et d'accumulation du savoir maintenue par les acteurs actuels - de même que par les bénéficiaires de ce patrimoine immatériel. Une partie de la pensée critique muséologique d'aujourd'hui est la reconnaissance même de la muséologie en tant quarène politique où la tradition est négociée et transformée.

Quelques-uns des contributeurs de ce numéro, regardant la muséologie depuis des périphéries du Nord mondialisé - le traditionnel centre de production du savoir - tirent avantage de quelques divergences avec la tradition pour proposer des interprétations innovantes face aux problèmes actuels. La muséologie expérimentale, telle qu'explorée par Melissa Aguilar Rojas dans son article, embrasse l'appropriation de l'espace muséographique avec de nouvelles expres- 
sions pratiques fondées sur l'expérience sensorielle des visiteurs. La relation entre la muséologie théorique et la méthode pratique pour les approches expérimentales menées au musée est au cour des intérêts de Rojas. S'arrêtant sur le contexte de l'Amérique latine, elle souligne la manière selon laquelle la pensée critique associée à l'usage de nouvelles technologies peut créer des opportunités pour «pirater le circuit habituel d'information ». Pirater le musée, en l'expérimentant sous différentes formes et avec de nouvelles interactions sociales, peut être une manière innovante de recréer la tradition tout en multipliant d'autres alternatives pour le futur.

Un point central dans l'article de Rojas tient au fait que la muséologie expérimentale existe au-delà du musée - une déclaration qui peut être rapprochées de celles de plusieurs théoriciens de notre tradition. Dans une approche différente, Scarlet R. Galindo Monteagudo propose une interprétation de la muséologie sous l'angle de l'Actor Network Theory (ANT) de Bruno Latour et de la sociologie symétrique pour démontrer comment une micro-analyse du musée peut prendre en considération l'agency des humains et des non-humains au-delà de leur réalité physique immédiate. Ces études montrent, par exemple, comment les musées exercent leur rôle dans les sociétés, comment ils communiquent à leurs publics en créant des liens et en produisant un engagement social.

À la lumière de nouvelles valeurs partagées par les sociétés actuelles, il est possible de supposer qu'un musée est un espace relationnel, fondé localement et dépendant de la participation sociale, potentiellement défini comme post-colonial et post-national, non soumis à des constructions européo-centriques - comme Mellado \& Andrade le proposent dans leur analyse. Mais cet idéal quelque peu utopique en faveur d'une institution démocratique et décolonisée peut révéler certaines contradictions dans le présent. Si les musées sont post-coloniaux et post-nationaux par principe, nous ne pouvons ignorer l'importance des États pour assurer leur survie en période d'incertitudes économiques et de crises politiques. Dans la majorité des pays du monde, du Nord comme du Sud, une grande partie des collections de musées forme une part permanente du patrimoine national conservé par un État depuis les débuts de la Modernité.

L'existence actuelle des musées dépend amplement des manières dont nous transmettons le patrimoine culturel aujourd'hui, un processus qui est relié aux valeurs du présent mais qui peut aussi déterminer le futur des musées. En période d'incertitudes, qui se multiplient dans le secteur culturel, on peut se demander : comment les musées peuvent-ils s'adapter à un monde culturel dynamique sans se satisfaire du marché culturel qui développe des inégalités sociales et une répartition inéquilibrée du patrimoine à travers le globe ? Comment garantir une transmission du patrimoine culturel à tous dans un monde où la culture a un prix et que tous ne peuvent le payer? En d'autres termes, comment les musées et la muséologie peuvent-ils évoluer en vue du futur, sans contradiction avec les traditions et leurs engagements envers la société qui les définissent dans le présent? 
Les réflexions réunies dans les pages qui suivent peuvent aider à considérer des solutions créatives pour des futurs musées et muséologies. Une alternative possible est de trouver des voies pour rompre avec le passé tout en assurant sa transmission aux générations futures, qui y réfléchiront et produiront de nouvelles interprétations de leurs propres traditions, d'après leurs propres conceptions du temps. Ainsi, les musées doivent être l'expression à la fois de la tradition et de la transmission, en rendant visibles les tensions derrière les diverses interprétations du passé qui matérialise un futur susceptible d'être changé, contesté, réécrit au présent. C'est au sein de cet espace entre la tradition et la transmission que le passé peut être réinventé pour créer un meilleur futur.

\section{Références}

Augé, P. (Dir.). (1931). Larousse du XXe siècle. v. 4. Paris : Librairie Larousse, p.IO48.

Bancel, N., Bernault, F., Blanchard, P., Boubeker, A., Mbembe, A., \& Vergès, F. (20IO). Introduction: De la fracture coloniale aux ruptures postcoloniales. Dans Achille Mbembe et al., Ruptures postcoloniales. La Découverte, Cahiers libres, 2010, p. 9-34.

Bhabha, H. K. (1994). The location of culture. London and New York: Routledge.

Brulon Soares, B., \& Leshchenko, A. (2018). Museology in Colonial Context: A Call for Decolonisation of Museum Theory. ICOFOM Study Series, 46, 6I-79.

Cunha, M., \& Almeida, M. W. B. (2009) Populações tradicionais e conservação ambiental. Dans M. Cunha (Ed.), Cultura com aspas e outros ensaios (pp. 277-300). São Paulo: Cosac Naify.

Déclaration de Québec (1984). Principes de base d’une nouvelle muséologie, Adoptée par le r ${ }^{\text {er }}$ Atelier international Écomusée / Nouvelle muséologie, Québec, le I2 octobre, I984. Consulté le i 8 avril 2020 depuis http://www.minomicom.net/_old/signud/DOC\%20PDF/198402504.pdf

Desvallées, A. (1992). Présentation. Dans A. Desvallées, M.-O. de Bary, F. Wasserman (1992), Vagues, une anthologie de la nouvelle muséologie. (pp. 15-39). Macon et Savigny-le-Temple, W et Mnes, t.I.

Fabian, J. (2002 [1983]). Time and the other: how anthropology makes its object. New York: Columbia University Press.

Konaré, A. O. (1987). L'idée du musée. ICOFOM Study Series, I2, I5I-I55.

Mairesse, F. (2015). New trends in museology, ICOFOM Study Series, 43a, I3-15.

Mairesse, F. (20II). Musée. Dans A. Desvallées \& F. Mairesse (Dir.), Dictionnaire encyclopédique de muséologie (pp. 271-288). Paris: Armand Colin. 
Mignolo, W. D. (I995). La razón postcolonial: herencias coloniales y teorías postcoloniales. Revista Chilena de Literatura, 47, 9I-II4.

Pearce, S. (2010). The collecting process and the founding of museums in the sixteenth, seventeenth and eighteenth centuries. Dans S. Pettersson, M. Hagedorn-Saupe, T. Jyrkkiö, \& A. Weij (Ed.), Encouraging collections mobility. A way forward for museums in Europe (p.r2-33). Kaivokatu: Finnish National Gallery.

Pletsch, C. E. (198I). The Three Worlds, or the Division of Social Scientific Labor, Circa I950-I975. Comparative Studies in Society and History, $23(4)$, 565-590. Consulté le i8 avril 2020 depuis www.jstor.org/stable/I78394

Poulot, D. (Dir.). (1998). Patrimoine et modernité. Paris : L'Harmattan.

Quijano, A. (2000). Coloniality of Power, Eurocentrism and Latin America. Nepantla: Views from South, I(3), 533-580.

Rivière, G. H., \& Cuisenier, J. (I972). Le musée des arts et traditions populaires, Paris. Museum International, XXIV(3), I8I-I84.

Rússio, W. (I989). Muséologie et futurologie : esquisse d'idées. ICOFOM Study Series, 16, 219-226.

Smeds, K. (2019). Introduction. In K. Smeds (Ed.), The future of tradition in museology. Materials for a discussion. Paris: ICOFOM/ICOM.

Sofka, V. (Ed.). (I989). Forecasting - a museological tool? Museology and futurology / La prospective - un outil muséologique? Muséologie et futurologie. Preprints to the ICOFOM Symposium in The Hague, The Netherlands. ICOFOM Study Series, I6.

Varine, H. de (2017). L'écomusée singulier et pluriel. Un témoignage sur cinquante ans de muséologie communautaire dans le monde. Paris : L'Harmattan.

Yates, F. A. (1966). The Art of Memory. Chicago: University of Chicago Press.

Zavala, L. (20I2). Antimanual del museólogo. Hacia una museología de la vida cotidiana. México: UAM/INAH/Conaculta. 